\title{
Habilidades Sociais e o Envolvimento entre Pais e Filhos com Deficiência Intelectual
}

Social Skills and the Involvement Among Parents and Children with Intellectual Disabilities

Habilidades Sociales y el Envolvimiento entre Padres e Hijos con Deficiencia Intelectual

Alcides Cardozo \& Adriana Benevides Soares

Universidade Salgado de Oliveira
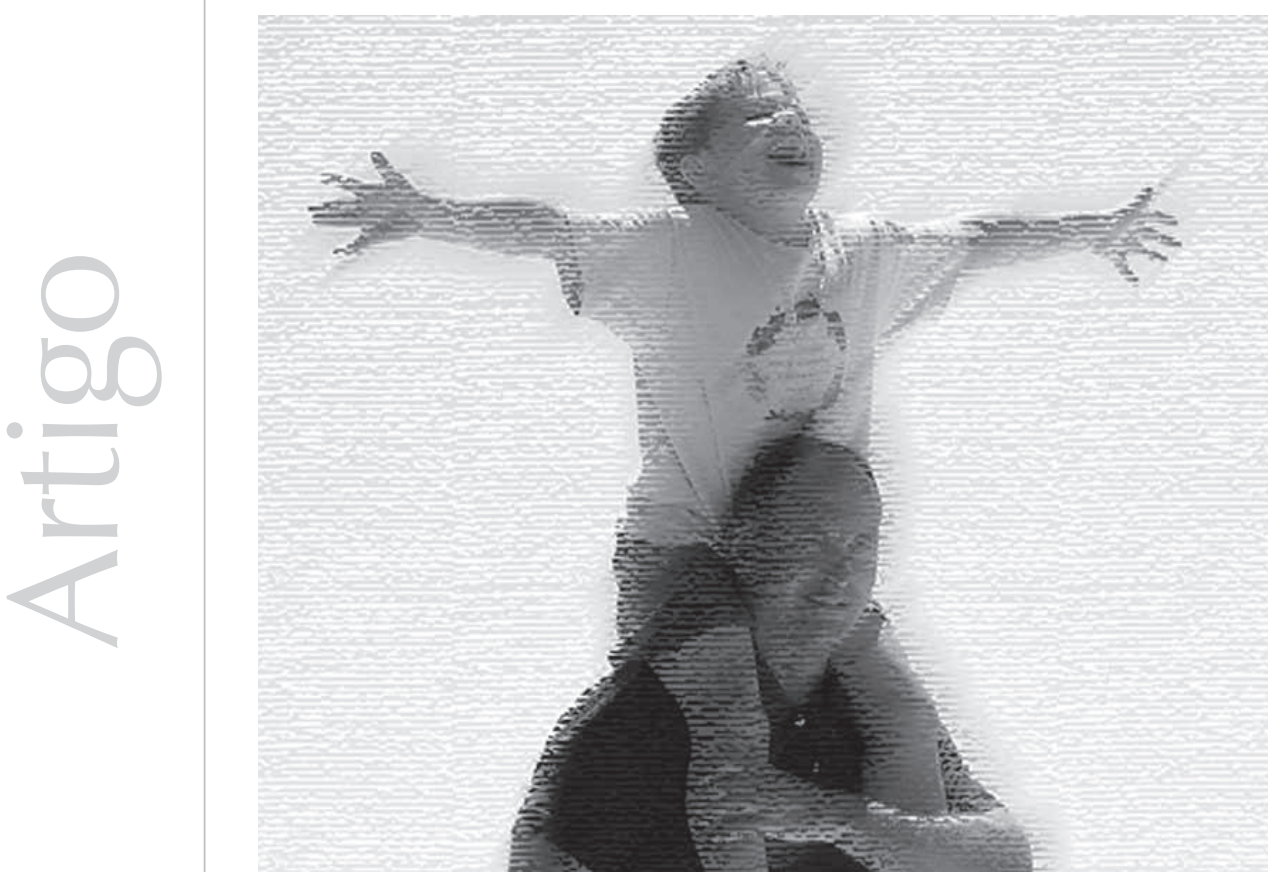
Resumo: Este artigo pretende abordar o tema das habilidades sociais relacionando-o ao envolvimento entre pais e filhos com deficiência intelectual. O portador de deficiência intelectual é um sujeito que necessita aprimorar suas relações interpessoais para desenvolver-se satisfatoriamente, de forma a conquistar mais autonomia e satisfação pessoal e melhor qualidade de vida. Muitos autores destacam a importância de um repertório adequado de habilidades sociais dos pais estabelecendo um ambiente familiar acolhedor e propício para o desenvolvimento pleno dos filhos. Esse ambiente pode influenciar na qualidade do envolvimento com os filhos, transformando esses pais em modelos de comportamento social e colaborando ainda para que os filhos tenham um desempenho social mais autônomo e inclusivo. Há, entretanto, o estresse e as variáveis sociodemográficas, que, com a chegada de um filho com deficiência, podem determinar a incompetência social desses pais para a conquista de espaços mais harmoniosos destinados a receber esse filho.

Palavras-chave: Habilidades sociais. Retardo mental. Relações pais-criança. Competência social.

Abstract: This paper aims to investigate the parent's social skills and the influence of these skills on the parents' attachment to their children with intellectual disability. The child who suffers from intellectual disability needs to improve his/her interpersonal relations in order to reach a suitable development that allows him/her to get more autonomy, happiness and consequently a better way of life. Several authors have underlined the importance of an adequate repository of social abilities from parents to set up a warm familiar environment, which is propitious for the development of their children. This environment would influence the quality of the attachment to their children, changing these parents in examples of social behaviors and, additionally, collaborating with the improvement of the social life of their children, allowing them to be more autonomous and inclusive. Nevertheless, stress and some social-demographic variables, with the advent of a handicapped child, can determine the social incompetence of the parents in order to provide harmonious places to accept this child.

Keywords: Social skills. Mental retardadion. Parent child relations. Social competence.

Resumen: Este artículo pretende abordar el tema de las habilidades sociales relacionándolo al envolvimiento entre padres e hijos con deficiencia intelectual. El portador de deficiencia intelectual es un sujeto que necesita mejorar sus relaciones interpersonales para desarrollarse satisfactoriamente, de forma a conquistar más autonomía y satisfacción personal y mejor calidad de vida. Muchos autores destacan la importancia de un repertorio adecuado de habilidades sociales de los padres estableciendo un ambiente familiar acogedor y propicio para el desarrollo pleno de los hijos. Ese ambiente puede influenciar en la calidad del envolvimiento con los hijos, transformando a esos padres en modelos de comportamiento social y colaborando también para que los hijos tengan un desempeño social más autónomo e inclusivo. Existe, no obstante, el estrés y las variables socio-demográficas, que, con la llegada de un hijo con deficiencia, pueden determinar la incompetencia social de esos padres para la conquista de espacios más harmoniosos destinados a recibir ese hijo.

Palabras clave: Habilidades sociales. Retraso mental. Relaciones padres-ninos. Competencia Social

Segundo Cia, Pereira, Del Prette e Del Prette (2006) situações interpessoais podem ocorrer em diversos contextos, como, por exemplo, o familiar, o de lazer, o escolar e o profissional. Em cada contexto, esperamse determinados desempenhos que, por sua vez, exigem um amplo repertório de habilidades sociais dos indivíduos (p. 74). Esse repertório, e mais especificamente o de habilidades sociais educativas dos pais, pode influenciar a intensidade e a qualidade do envolvimento destes com os filhos portadores de deficiência intelectual e, em consequência, o tipo de prática educativa que adotam na relação com eles. Por outro lado, os pais que apresentam dificuldades interpessoais podem prejudicar a qualidade desse relacionamento, além de oferecer modelos inadequados de desempenho social (Barros, 2008, p. 48, Bolsoni-Silva, Del Prette, \& Del Prette, 2000; Del Prette \& Del Prette, 2002).

Quando uma criança fica à mercê de comportamentos pouco construtivos de pais ou sem o envolvimento afetivo destes, isso poderá constituir prejuízo para o desenvolvimento da criança e aumentar sua vulnerabilidade nos diversos contextos sociais. Em contrapartida, pais socialmente habilidosos, que estabelecem um ambiente familiar acolhedor, organizam ambientes favoráveis aos mecanismos de resiliência e de proteção diante de fatores ameaçadores aos quais, usualmente, as 
crianças estão expostas (Del Prette \& Del Prette, 2005; Yunes, 2003).

Procurando abordar a temática das habilidades sociais de pais e o envolvimento destes com os filhos portadores de deficiência intelectual em suas diversas dimensões e aspectos teóricos, conceituais e práticos, este estudo pretende discorrer sobre as características da deficiência intelectual e sobre a importância da promoção das habilidades sociais desses indivíduos pelos seus pais a fim de desenvolver comportamentos mais adequados que permitam melhor satisfação pessoal.

\section{Características da deficiência intelectual}

O termo deficiência intelectual tem sido objeto de controvérsia na literatura especializada. A definição da Organização Mundial de Saúde (CID-10, 1993) faz referência genérica a aspectos funcionais colocandoos como indicadores ou consequências do funcionamento intelectual rebaixado. Esse funcionamento é percebido como o núcleo da própria definição e do critério diagnóstico. (Barbosa e Del Prette, 2002, p.1). Para se ter um diagnóstico definitivo, segundo a Classificação Internacional de Doenças, na sua 10a. revisão (CID-10, 1993), deve-se observar um nível reduzido de funcionamento intelectual, o que resultará em capacidade diminuída para se adaptar às exigências diárias do contexto social normal.

O DSM-IV-TR (2003), Diagnostic and Statistical Manual of Mental Disorders, muito embora destaque uma etiologia orgânica, adiciona critérios funcionais e psicométricos na caracterização da deficiência intelectual, enfatizando os aspectos funcionais, ao definir e especificar áreas de conduta adaptativa como parte dos critérios diagnósticos. Essa definição orienta na verificação do funcionamento intelectual significativamente inferior à média, dos deficits ou comprometimentos concomitantes ao funcionamento adaptativo em pelo menos duas das seguintes áreas: comunicação, cuidados pessoais, vida doméstica, habilidades sociais/interpessoais, uso de recursos da comunidade, independência, habilidades acadêmicas, trabalho, lazer, saúde e segurança. Além disso, para a caracterização da deficiência intelectual, é necessário o início anterior aos 18 anos.

Kaplan, Sadock e Greb (1977) relatam que até pouco tempo atrás, tanto o termo deficiência mental quanto o termo retardo mental eram usados de modo intercambiável, quando então a American Association on Intellectual and Developmental Disabilities (2010) escolheu deficiência intelectual como o preferível. A AAIDD (2010) vem liderando o campo de estudos sobre deficiência intelectual, definindo conceituações, classificações, modelos teóricos e orientações de intervenções em diferentes áreas. Embora secular e influenciando sistemas de classificação internacionalmente conhecidos como o DSM-IV-TR e a CID-10, a AAIDD não é conhecida no Brasil como são os outros dois sistemas. Esse fato talvez se deva à pouca divulgação de suas produções entre especialistas e pesquisadores brasileiros. $\mathrm{O}$ atual modelo proposto pela AAIDD traduz uma concepção multidimensional, funcional e bioecológica de deficiência intelectual, adicionando sucessivas inovações e reflexões teóricas e empíricas em relação aos seus modelos anteriores. Luckasson et al (2002, p.43) apresenta a seguinte definição de deficiência intelectual: incapacidade caracterizada por limitações significativas, tanto no funcionamento intelectual quanto no comportamento adaptativo e está expressa nas habilidades práticas, sociais e conceituais, originando-se antes dos 18 anos de idade. Observa-se, nessa definição, que a deficiência intelectual não representa um atributo da pessoa, mas um estado particular de funcionamento (Almeida, 2004). 
A concepção multidimensional da Associação (citado em Carvalho e Maciel, 2003) explica a deficiência intelectual em cinco dimensões, que envolvem aspectos relacionados à pessoa, ao seu funcionamento individual no ambiente físico e social, ao contexto e aos sistemas de apoio. Uma dessas dimensões dá importância ao comportamento adaptativo, que se define como o conjunto de habilidades conceituais, sociais e práticas adquiridas pelo indivíduo para fazer face às necessidades da vida diária; as habilidades conceituais estão relacionadas aos aspectos acadêmicos, cognitivos e de comunicação; as habilidades sociais estão relacionadas à competência social exemplificada pela responsabilidade, autoestima, habilidades interpessoais, credulidade e ingenuidade, observância de regras, normas e leis; as habilidades práticas estão relacionadas ao exercício da autonomia, como: alimentar-se, arrumar a casa, deslocarse de maneira independente e utilizar meios de transportes. Uma outra dimensão que se considera também importante é a relativa participação, a interação e os papéis sociais em se que ressalta a importância da vida comunitária, que se refere à avaliação das interações sociais e dos papéis vivenciados pela pessoa bem como à sua participação na comunidade em que vive. São consideradas as condições em que a pessoa vive e sua qualidade de vida.

Segundo Del Prette e Del Prette (2002), a adoção de critérios funcionais de diagnósticos da deficiência intelectual revela uma preocupação com a reversão das dificuldades desses indivíduos entendidas como necessidades educacionais especiais. Essa noção remete a uma perspectiva educacional em termos do compromisso não só da família como também da escola, com objetivos socialmente relevantes para essa clientela e com planejamento de intervenção social e educativa, mostrando, portanto, a questão do relacionamento interpessoal desses indivíduos e as habilidades requeridas para isso. (Barbosa e Del Prette, 2002, p. 1).

\section{A importância das habilidades sociais}

As relações interpessoais, base da vida em sociedade, são uma parte essencial da atividade humana. Praticamente todas as teorias de desenvolvimento abordam a questão da socialização e da importância das interações e relações sociais como indicadores de saúde mental e de desenvolvimento. As habilidades sociais estão sempre nos contextos e, com a crescente complexidade das demandas sociais, quer profissionalmente, quer pessoalmente, há uma exigência cada vez maior dessas habilidades.

Carneiro e Falcone (2004) definem o indivíduo socialmente habilidoso como aquele que é capaz de lucrar com maior frequência, realizar o mínimo de tarefas desagradáveis e desenvolver ainda relacionamentos benéficos e produtivos. Assim, a emissão de comportamentos socialmente habilidosos faz aumentar a satisfação pessoal e, em consequência, levar a melhoria na qualidade de vida.

Segundo Caballo (1996), as condutas socialmente habilidosas constituem uma série de comportamentos emitidos em um contexto interpessoal que refletem os sentimentos, atitudes, desejos, opiniões e direitos do indivíduo de um modo adequado à situação, com o respeito por essas condutas nos outros e a disposição para solucionar os problemas imediatos daquela situação, além da minimização da possibilidade de ocorrência de futuros problemas.

No relato de Molina e Del Prette (2006), as habilidades sociais podem também constituir necessidades especiais educativas para qualquer criança na medida em que os deficits em relação aos parâmetros de normalidade, ou seja, ao repertório minimamente desejável em dado contexto social e cultural, dificultam ou impedem seu funcionamento adaptativo 
em termos de independência pessoal, responsabilidade social e qualidade das relações. Há necessidade, portanto, que os pais, figuras centrais na educação dos filhos dentro do primeiro microssistema, que é o familiar, estejam atentos desde cedo às suas práticas quanto às habilidades sociais aplicáveis à educação das crianças (Silva, 2000). Essa última autora afirma que habilidade social educativa de dialogar com os filhos, por exemplo, é o alicerce para o desenvolvimento de todas as demais habilidades sociais educativas. Elas auxiliam os pais a mostrarem os padrões de comportamentos, de valores e de normas de cultura para os filhos.

Nas últimas décadas, as habilidades sociais têm obtido um importante papel no estudo de pessoas com distúrbios de aprendizagem, com atraso no desenvolvimento e com deficiência intelectual. Os estudos sobre competência social dessas pessoas, segundo Del Prette e Del Prette (2005), apontam dificuldades na estruturação de frases, deficits nos componentes da comunicação não verbal e baixa competência na habilidade para reformulação da própria fala. Ainda segundo esses autores, pesquisas recentes com adultos portadores de deficiência intelectual constataram falhas em diversas áreas, principalmente nas habilidades assertivas de lidar com críticas, de recusar e discordar e nas relacionadas a dificuldade na emissão de componentes expressivos, verbais e não verbais.

Rosin-Pinola, Del Prette e Del Prette (2007), ao compararem o desempenho social de alunos com alto e baixo rendimento e deficientes intelectuais, constataram que, na avaliação do professor, tanto o grupo de baixo rendimento quanto o dos deficientes apresentam dificuldades acadêmicas e baixos escores relativos a habilidades sociais de responsabilidade, autocontrole e cooperação entre os pares. O grupo portador de deficiência apresenta ainda baixos escores nos itens assertividade e autodefesa, apresentando, segundo os autores, uma desvantagem de aquisição. Diferencia-se dos outros grupos também no que diz respeito à briga com os outros, baixa autoestima, ameaça ou intimidação, apresentando problemas de comportamento externalizantes.

Riches (1996) propõe que se dê atenção especial às habilidades sociais exigidas na vida diária de pessoas com deficiência intelectual. Durante muito tempo, foi dada ênfase às habilidades específicas, como treino de ir ao toalete, vestir-se ou alimentarse de forma independente ou mesmo habilidades essenciais para a sobrevivência em comunidade e para a vida independente, como manejo de dinheiro e habilidades domésticas. Segundo a autora, é preciso também dar atenção aos programas dirigidos aos processos de interação social de forma que estejam fundamentados no entendimento do encontro entre as pessoas. Nesse sentido, avaliar e promover o envolvimento dos pais com os filhos pode ser a mola propulsora do desencadeamento de melhor desempenho interpessoal desses filhos. Os pais podem servir de modelos comportamentais para os filhos, e uma boa relação entre pais e filhos que desenvolva vínculos afetivos pode propiciar comportamentos mais competentes, gerando mais autonomia e possibilidades de inclusão social.

\section{O envolvimento entre pais e filhos com deficiência intelectual}

A família, desde tempos longínquos, corresponde a um grupo social que exerce marcada influência sobre a vida das pessoas, e tem sido apontada como uma das únicas possibilidades de desenvolvimento social e emocional para uma criança. A estrutura familiar, quando em bom funcionamento, demonstrando uma clara separação de 
Segundo

Paniagua

(2004), ter um

filho é um dos

acontecimentos

mais vitais para

um ser humano.

Os vínculos

afetivos entre

pais e filhos

normalmente

são tão intensos

como as

emoções que

se põem em

jogo. Um filho é

sempre fonte de

ilusões ou medos. gerações oferece a cada componente desses subsistemas a experiência de participar de um ambiente seguro e acolhedor (Terra dos Homens, 2002). Neste sentido, a harmonia, a qualidade do relacionamento entre pais e a qualidade do relacionamento entre pais e filhos são aspectos importantes que irão exercer influência direta na sua personalidade e no seu sucesso escolar (Tállon, Ferro, Gomes, \& Parra, 1999). Assim, a relação entre pais e filhos é a que apresenta o vínculo mais forte dentro do contexto familiar, pois, segundo Romanelli (1997), está ligada à reprodução da família em sentido mais amplo, englobando a reprodução biológica e, principalmente, a reprodução social.

Romanelli (1998, 2003), em pesquisa realizada com pais e filhos, verificou que famílias de classes médias baixas mudaram suas percepções quanto ao papel do genitor com os filhos. O pai concebe a existência de maior proximidade na relação, com maior expressão de afeto pelos filhos e disponibilidade para dialogar. A autoridade deixa de ser exercida para impor a vontade, e os filhos podem, então, expressar suas reivindicações. Os pais procuram avaliar as peculiaridades dos filhos apreendendo e procurando aceitar aquilo que os constitui únicos. Os pais avaliam e explicitam suas preocupações com a escolarização dos filhos, pois isso resultará em maior segurança e garantia de seus direitos individuais.

Em uma perspectiva social abrangente, é importante compreender o processo de desenvolvimento de crianças com necessidades especiais educativas por meio da análise de variáveis da pessoa, do processo interacional e do contexto imediato e mediato do âmbito da família (Sigolo, 2004). O contexto familiar deve ser considerado de importância crucial para o desenvolvimento infantil, podendo atuar como fonte de recursos e mecanismos protetores para lidar com condições adversas. De maneira geral, os pais expressam níveis elevados de afeto positivo e apresentam razoável disposição para interagir.

Segundo Paniagua (2004), ter um filho é um dos acontecimentos mais vitais para um ser humano. Os vínculos afetivos entre pais e filhos normalmente são tão intensos como as emoções que se põem em jogo. Um filho é sempre fonte de ilusões ou medos. O fato de que ser pai seja algo habitual não significa que seja fácil, pois quando o filho tem alguma dificuldade, tudo pode se tornar muito difícil. Por outro lado, Nunes (2003), em seus estudos sobre as famílias com filhos com deficiência, descreve os conflitos presentes nos vínculos e os indicadores de risco no meio familiar. Essa autora concluiu que esses conflitos não surgem do resultado direto da deficiência, mas da adaptação ou não a essa nova realidade.

Desde o momento em que os pais ficam sabendo da existência de uma deficiência, há muita preocupação com o presente e com o futuro da criança que irá acompanhá-los por toda a vida. Ao longo do desenvolvimento da criança, os pais terão de decidir sobre tratamentos médicos, opções educativas, enfim, sobre o aumento de dedicação que, em geral, requer um filho com necessidades especiais. Muitas vezes, a criança com deficiência irá necessitar de muito mais cuidados físicos, assim como de mais tempo de interação e mais situações de jogo ou estudo compartilhado. O desenvolvimento de programas de estimulação desde cedo, as atividades de lazer e o reforço familiar ao longo da escolarização representa, para os pais, um grande esforço pessoal.

Góes (2006) constata que, para os pais, a deficiência intelectual na criança significa a perda do filho idealizado, e que aqueles têm dificuldade em encontrar sinais que se ajustem às suas representações do filho 
ideal. Além disso, existem, no ambiente cultural, representações sociais preenchidas de qualidades desfavoráveis em relação à deficiência intelectual. Os pais acumulam crenças geralmente negativas, e estas são referências iniciais para a construção de suas representações sobre o filho portador de necessidades especiais.

Glat e Duque (2003), em uma pesquisa qualitativa com dezesseis pais de filhos com necessidades especiais, ao analisarem a dinâmica de ações e relações familiares a partir de seus depoimentos, concluíram que a preocupação com a incerteza do futuro de seus filhos foi um ponto relevante nesse estudo. As autoras viram que essa preocupação faz com que eles se esforcem para dar aos filhos uma educação que possa, principalmente, desenvolver habilidades que garantam maior independência e autonomia possível na vida adulta. Nesse estudo, as autoras concluíram também que, apesar de os pais viverem suas angústias, desespero e depressão no contato íntimo e diário com seus filhos, eles tiveram inúmeras oportunidades de compensação e conseguiram superar as crises, de acordo com sua maneira de ser, amando e convivendo com os filhos apesar de todas as dificuldades.

Hanson (2003) estudou famílias em que filhos com síndrome de Down participaram de um programa de intervenção na infância, que foi reavaliado vinte e cinco anos depois. Os dados mostraram que os pais percebem as características positivas da criança, desde o fato de ser considerada uma benção até as experiências prazerosas dos pais ao verificarem, no dia a dia, as aquisições dos filhos com síndrome de Down.

Segundo Cia, d'Affonseca e Barham (2004), são poucos os estudos que nos permitem saber qual é a qualidade do relacionamento entre pais e filhos no Brasil. Além disso, o papel do pai se encontra em fase de mudanças, sendo inúmeras as famílias em que ambos (mãe e pai) trabalham fora, o que tem levado à redefinição do papel paterno. Essas autoras estudaram o impacto da qualidade do relacionamento entre pai e filho sobre o desempenho acadêmico de crianças escolares, em amostra de cinquenta e oito pais e seus filhos que cursavam os anos finais do ensino fundamental. Puderam verificar que, quanto maior a frequência de comunicação entre pai e filho e a participação dos pais nas atividades escolares, culturais e de lazer, maior a pontuação das crianças em escrita e leitura e maior o seu desempenho acadêmico.

Uma outra pesquisa que teve como objetivo comparar e correlacionar indicadores do repertório de habilidades sociais e do envolvimento dos pais na educação dos filhos (Cia, d'Affonseca, \& Barham, 2006) revela que os relatos realizados pelos cônjuges indicam atividades diferenciadas. Os pais, por exemplo, se ocupavam com maior frequência em proporcionar lazer fora de casa aos filhos, e as mães, em estabelecer horário de deitar e em controlar a higiene. Essa diferença de participação e de envolvimento dos pais (mãe e pai) nos cuidados com os filhos é semelhante aos dados das pesquisas de Bertolini (2002), em que há uma divisão razoavelmente estruturada de atividades, na qual o homem se ocupa da parte social e de lazer e as mães, dos cuidados diários da casa. Segundo essa autora, nesse estudo em que verificaram as relações entre o trabalho da mulher e a dinâmica familiar, alguns homens afirmam que, sensibilizados com o acúmulo de atividades das companheiras dentro e fora do lar, estão dispostos a dar sua parcela de contribuição espontaneamente. O impacto dessas mudanças para o desenvolvimento infantil somente poderá ser conhecido a partir de estudos que avaliem a interação dos pais com seus filhos. 


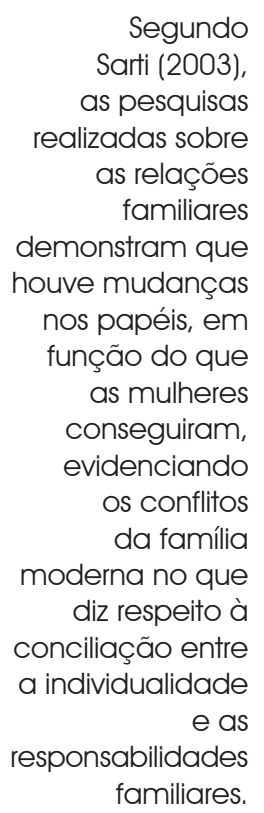

Cardozo e Soares (2010) investigaram se as habilidades sociais dos pais de crianças com deficiência intelectual influenciaram o seu envolvimento na educação dos filhos. Foram comparados indicadores do repertório de habilidades sociais e do envolvimento dos pais na educação dos filhos, e foram também comparados mães e pais nos dois conjuntos de medida. A hipótese de que as mães seriam mais envolvidas na educação dos filhos com deficiência intelectual do que os pais foi corroborada. Os resultados apontaram maior participação das mães na educação, na participação das atividades escolares, no lazer e nas atividades culturais e nos cuidados com os filhos. Demonstrou-se que, apesar de os pais (homens) apresentarem um repertório de habilidades sociais um pouco mais enriquecido, isso não foi relevante para que se envolvessem mais com os filhos.

Segundo Dessen e Costa (2005), em um trabalho com crianças com desenvolvimento típico, o relacionamento marital estaria sendo apontado como fator importante para a qualidade das relações que os pais mantêm com os filhos. Segundo as autoras, a convivência entre cônjuges, quanto às formas de comunicação e estratégias para resolver os problemas, estariam influenciando a criação de estilos parentais de cuidados dos filhos e a qualidade dessas relações. No que tange às situações de conflito, esses autores relatam que mães insatisfeitas tendem a compensar os filhos sendo mais responsivas e envolvendo-se mais com suas crianças. Por outro lado, pais emitem condutas negativistas e intrusivas em relação aos filhos, afastando-se do convívio mais direto, apesar de viverem sob o mesmo teto. É possível que, no caso de pais e mães de filhos com deficiência intelectual, isso possa ocorrer, principalmente, com relação ao estresse vivenciado pelo pai, oriundo às vezes de dificuldades financeiras, e pelo fato de ter um filho com deficiência intelectual. Afinal, os sentimentos e as representações familiares que existiam anteriormente ao nascimento desse filho se deterioram, gerando uma crise de identidade nesse pai. Por outro lado, o envolvimento das mães de filhos com deficiência intelectual, principalmente com relação aos cuidados, leva-as a caminhar à procura de tratamento para os filhos. Miltiades e Pruchno (2001) realizaram um estudo com mães de filhos adultos com deficiência e chegaram à conclusão que essas mulheres continuam vivendo situações de cuidado e de responsabilidades pela vida dos filhos, assumindo um papel vitalício de cuidadoras. Estão sempre procurando manter as diversas formas de tratamento, em infindáveis negociações com as instituições de reabilitação ou redes de apoio. Em função dessa demanda, pode-se supor que tenham adquirido habilidades de traquejo na conversação, o que supõe conhecimento das regras e normas de relacionamento.

Segundo Sarti (2003), as pesquisas realizadas sobre as relações familiares demonstram que houve mudanças nos papéis, em função do que as mulheres conseguiram, evidenciando os conflitos da família moderna no que diz respeito à conciliação entre a individualidade e as responsabilidades familiares. Segundo a autora, nas famílias que possuem condições econômicas deficitárias, esse conflito é menor em decorrência dos vínculos familiares que se sobrepõem aos indivíduos. Percebe-se, no entanto, que, apesar dos avanços sociais da mulher, ela ainda ocupa uma posição de maior subordinação nas relações familiares, que, em geral, permanecem com um padrão tradicional e hierárquico de funcionamento.

Ainda que os papéis de mães e pais continuem se diferenciando segundo uma tradição histórica cultural já antiga, em que pais têm o papel de provedores e mães de cuidadoras e que, ainda hoje, sejam mais envolvidas com os filhos, quando se trata de pais e mães de portadores de deficiência intelectual, essas manifestações de habilidade e envolvimento na educação dos filhos têm se mostrado essenciais para o desenvolvimento dos filhos. À medida que os pais são 
habilidosos, servem de modelos referenciais para seus filhos no desenvolvimento e no aprimoramento de comportamentos também mais habilidosos. O envolvimento com os filhos os ajuda a descortinar um vínculo afetivo que lhes proporciona mais segurança no relacionamento interpessoal e, em consequência, melhor desempenho de comportamentos competentes socialmente, ou seja, mais autonomia e qualidade de vida.

\section{Alcides Cardozo}

Mestre em Psicologia, Psicólogo exercendo atividades com vistas a orientação familiar, avaliação, orientação e tratamento da pessoa com deficiência e transtornos mentais, bem como visitas domiciliares e institucionais. Participação como colaborador nos projetos de pesquisa da UNIVERSO - Universidade Salgado de Oliveira, 1) O repertório de habilidades sociais de professores e sua representação social.2) Cognição, habilidades sociais e praticas pedagógicas, Rio de Janeiro - RJ - Brasil.

E-mail: alcidescardozo@yahoo.com.br

\section{Adriana Benevides Soares}

Doutora em Psicologia Cognitiva pela Universidade de Paris XI, Professora adjunta da Universidade do Estado do Rio de Janeiro e professora titular da Universidade Salgado de Oliveira, Rio de Janeiro - RJ - Brasil.

E-mail: adribenevides@gmail.com

Endereço para envio de correspondência:

Universidade Salgado de Oliveira

R. Marechal Deodoro 263 Centro - Rio de Janeiro - RJ -Brasil

CEP: 24030-060 


\section{Referências}

Almeida, M. A. (2004). Apresentação e análise das definições de deficiência mental propostas pela AAMR - Associação Americana de Retardo Mental de 1908 a 2002, Revista de Educação PUC-Campinas, 16, 33-48.

Bolsoni-Silva, A. T., Del Prette, A., \& Del Prette, Z. A. P. (2000). Relacionamento pais-filhos: um programa de desenvolvimento interpessoal em grupo. Psicologia Escolar e Educacional, 3(3), 203-215.

Bertolini, L. B. A. (2002). Relação entre o trabalho da mulher e a dinâmica familiar (2a ed.). São Paulo: Vetor.

Barbosa, M. V. L.; Del Prette, Z.A. (2002). Habilidades sociais em alunos com retardo mental: análise de necessidades e condições. Revista do Centro de Educação, 20, 1-19.

Barros, S. K. S. N. (2008). Treinamento de Habilidades sociais para pais de crianças com queixas escolares, Dissertação de mestrado, Programa de Pós-Graduação em Educação Especial, UFSCAR.

Caballo, V. E. (1996). Manual de técnicas de terapia e de modificação de comportamento. São Paulo: Santos.

Cardozo, A., \& Soares, A. B. (2010). A influência das habilidades sociais no envolvimento dos pais e filhos com retardo mental. Aletheia, 31, 39-53.

Carneiro, R. S., \& Falcone, E. M. O. (2004). Um estudo das capacidades e deficiências em habilidades sociais na terceira idade. Psicologia em Estudo, 9(1), 119-126.

Cia, F., d'Affonseca, S. M., \& Barham, E. J. (2004). A relação entre o envolvimento paterno e o desempenho acadêmico dos filhos. Paidéia: Cadernos de Psicologia e Educação, 14(29), 277-286.

Cia, F., Pereira, C. S., Del Prette, Z. A. P. \& Del Prette, A. (2006). Habilidades sociais parentais e o relacionamento entre pais e filhos. Psicologia em Estudo, 11(1), 73-81.

Carvalho, E. N. S.; Maciel, D. M. M. A. (2003). Nova concepção de deficiência mental segundo a American Association on Mental Retardation-AAMR: sistema 2002, Temas em Psicologia, 11, 2, 147-156.

CID-10 (1993). Classificação de transtornos mentais e de comportamento da CID-10: descrições clínicas e diretrizes diagnósticas. Porto Alegre: Artes Médicas.

Del Prette, Z. A. P., \& Del Prette, A (2002). Psicologia das habilidades sociais: terapia e educação (3a ed.). Petrópolis, RJ: Vozes.

Del Prette, Z. A. P., \& Del Prette, A. (2004). Psicologia das relações interpessoais: vivências para o trabalho de grupo (3a ed.). Petrópolis, RJ: Vozes.

Del Prette, Z. A P., \& Del Prette, A. (2005). Psicologia das habilidades sociais na infância: teoria e prática. Petrópolis, RJ: Vozes.

Dessen, M. A., \& Costa Jr., A. L. (2005). A ciência do desenvolvimento humano: tendências atuais e perspectivas futuras. Porto Alegre: Artes Médicas.

Diagnostic and Statistical Manual of Mental Disorders - DSM-IV (2003). Referência rápida aos critérios diagnósticos (4a. ed.). Porto Alegre: Artmed.

Glat, R., \& Duque, M. A. T. (2003). Convivendo com crianças especiais: o olhar paterno. Rio de Janeiro: Sette Letras.

Goés, F. A. B. (2006). Um encontro inesperado: os pais e seu filho com deficiência mental. Psicologia: Ciência e Profissão, 26(3), 450-461

Hanson, M. (2003). Twenty-five years after early intervention: A follow-up of children with down syndrome and their families. Infants and Young Children, 16(4), 354-365.
Kaplan, H. I. Sadock, B. J., \& Grebb, J. A. (1997). Retardo mental. In H. I. Kaplan, B. J. Sadock \& J. A. Grebb, Compêndio de psiquiatria: ciências do comportamento e psiquiatria clínica (7a ed. pp. 955- 969,). Porto Alegre: Artes Médicas.

Luckasson, R., Borthwick-Duffy, S., Buntinx, W. H. E., Coulter, D. L., Caig, E. M., Reeve, A., Shallock, R. I., Snell, M. E., Spitalnik, D. M. E.,Spreat, S., \& Tessé, M. J. (2002) Mental retardation: definition, classification and systems of supports (10 ${ }^{\mathrm{a}}$ edição), Washington (DC): American Association on Mental Retardation.

Miltiades, H. B., \& Pruchno, R. (2001). Mothers of adults with developmental disability: Change over time. American Journal of Mental Retardation, 106(6), 548-561.

Molina, R. C. M., \& Del Prette, Z. A. P. (2006). Funcionalidade da relação entre habilidades sociais e dificuldades de aprendizagem. Psico-USF, 11(1), 53-63.

Nunes, B. (2003). La família com um hijo com discapacidad: sus conflictos vinculares, Archives Argentinian of Pediatry, Buenos Aires: Sociedad Argentina de Pediatria, 101 (2), 133-142.

Paniagua, G. (2004). As famílias de crianças com necessidades educativas especiais. In C. Coll, A. Marchesi, \& J. Palacios, (Eds.), Desenvolvimento psicológico e educação: transtornos de desenvolvimento e necessidades educativas especiais (pp.330346). Porto Alegre: Artes Médicas.

Riches, V. (1996). Everyday social interaction. A program for people with desabilities. Baltimore: Paul H. Books Publishing Co.

Schalock, Robert L.; Borthwick-Duffy, Sharon A.; Bradley, Valerie J.; Buntinx, Wil H. E.; Coulter, David L.; Craig, Ellis M.; Gomez, Sharon C.; Lachapelle, Yves; Luckasson, Ruth; Reeve, Alya; Shogren, Karrie A.; Snell, Martha E.; Spreat, Scott; Tasse, Marc J.; Thompson, James R.; Verdugo-Alonso, Miguel A.; Wehmeyer, Michael L.; \& Yeager, Mark H. (2010). Intellectual disability: definition, classification, and systems of supports (11a ed.). Washington, DC, USA: AAMR.

Romanelli, G. (1997). Famílias de classes populares: socialização e identidade masculina. Cadernos de Pesquisa NEP, 1-2, 25-34.

Romanelli, G. (1998). O relacionamento entre pais e filhos em famílias de camadas médias. Paidéia, 8(14), 123-136.

Romanelli, G. (2003). Paternidade em famílias de camadas médias. Estudos e Pesquisas em Psicologia, 3(2), 79-95.

Rosin-Pinola, A. R., Del Prette, Z. A. P., \& Del Prette, A. (2007). Habilidades sociais e problemas de comportamento de alunos com deficiência mental, alto e baixo rendimento acadêmico, Revista Brasileira de Educação Especial, 13(2) , 239-256.

Sarti, C. A. (2003). A família como espelho: um estudo sobre a

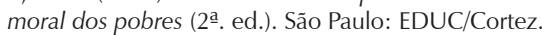

Sigolo, R. R. L. (2004). Favorecendo o desempenho infantil: ênfase nas trocas interativas no contexto familiar. In E. G. Mendes, M. A. Almeida, \& L. C. A. Willians (Eds.), Temas em educação especial: avanços recentes (pp. 189-195). São Carlos, SP: EDUFSCAR

Silva, A. T. B. (2000). Problemas de comportamento e comportamentos socialmente adequados: sua relação com as habilidades sociais educativas de pais. Dissertação mestrado, Universidade Federal de São Carlos, São Carlos, SP.

Tallón, M. A., Ferro, M. J., Gomes, R., \& Parra, P. (1999). Evaliación del clima familiar en una muestra de adolescentes. Revista de Psicologia Geral y Aplicada, 4, 451- 462.

Terra dos Homens. (2002). Trabalho social com família. Rio de Janeiro: Booklink Publicações.

Yunes, M. A. M. (2003). Psicologia positiva e resiliência: o foco no indivíduo e na família. Psicologia em Estudo, 8, 75-84. 